\title{
649 EFFEROCYTOSIS DRIVES MYELOID INFLAMMASOME SIGNALING AND GASDERMIN D INDEPENDENT SECRETION OF IL-1 $\beta$ TO PROMOTE TUMOR GROWTH
}

${ }^{1}$ Sohini Roy*, ${ }^{2}$ Cara Lang, ${ }^{3}$ Yu Wang, ${ }^{2}$ Diana Graves, ${ }^{2}$ Xu Yaomin, ${ }^{2}$ Young Kim. ${ }^{1}$ VUMC, Nashville, TN, United States; ${ }^{2}$ Vanderbilt University, Antioch, TN, United States; ${ }^{3}$ Vanderbillt University, Nashville, TN, United States

Background Inflammation has long been associated with different stages of tumorigenesis as well as response to therapy. A key signaling pathway in this context is the casp- 1 inflammasome. However, to date, its role in cancer has been contradictory and context dependent. We previously reported myeloid casp-1 can promote tumor growth in T cell independent manner. However, the regulatory mechanism that drives the myeloid intrinsic inflammasome signaling in the context of tumor growth remains largely unknown.

Methods In order to gain finer details about the inflammasome pathway components in the different myeloid clusters, we analyzed tumor and blood samples from head and neck cancer patients using bulk as well as $10 \mathrm{X}$ single cell sequencing platforms. For in vivo tumor studies, genetically engineered preclinical mice models were used. For in vitro functional studies, cells were isolated from mice or human tumors/blood and differentiated to either MDSC or macrophages and subjected to various assays.

Results Our bulk sequencing of myeloid cells isolated from treatment naïve head and neck tumors revealed an enrichment for inflammasome genes. Unbiased pathway analysis of tumor infiltrating myeloid cells compared to matched peripheral blood monocytes revealed IL-1 $\beta$ signaling to be significantly altered in the tumor myeloids. In our single cell transcriptomic sequencing dataset on human head \& neck carcinoma with matched peripheral blood monocytes, we observed similar elevated inflammasome transcriptomic activity within specific clusters of tumor-infiltrating macrophages and myeloid derived suppressor cells. Interestingly, distinct inflammasome sensor genes, specifically NLRP3, had distinct co-expressions with IL-1 $\beta$ in specific myeloid subsets within the TME. Our data also indicates that myeloid-intrinsic caspase-1 signaling paradoxically increased tumor infiltrating myeloid cell survival without significant intratumoral trafficking into the tumor. When we explored the TME regulatory factors that regulate intratumoral myeloid inflammasome signaling, we found that NLRP3 dependent inflammasome signaling and IL-1 $\beta$ production promotes tumor growth in a Gasdermin D independent mechanism. Mechanistically, we show that efferocytosis of dying tumor cells by myeloid cells in the TME directly activates NLRP3 dependent inflammasome signaling and IL-1 $\beta$ production in myeloid cells to promote tumor growth rate.

Conclusions To our knowledge, we are the first to attribute the tumor supporting role of myeloid inflammasome signaling to efferocytic clearance of apoptotic debris in the tumor microenvironment. Our study thus opens an enticing option of novel therapeutic modality for treatment of solid tumors in future.

Ethics Approval All experimental procedures were approved by the Institutional Review Board of Vanderbilt University Medical Center (IRB: 170172).

http://dx.doi.org/10.1136/jitc-2021-SITC2021.649 